\title{
Optimized cross-linking mass spectrometry for in situ interaction proteomics
}

Zheng Ser ${ }^{1,2}$, Paolo Cifani ${ }^{1}$, Alex Kentsis ${ }^{1,2,3,4 *}$

${ }^{1}$ Molecular Pharmacology Program, Sloan Kettering Institute, Memorial Sloan Kettering Cancer Center, New York, NY

2 Tri-Institutional PhD Program in Chemical Biology, Memorial Sloan Kettering Cancer Center, New York, NY

${ }^{3}$ Department of Pediatrics, Memorial Sloan Kettering Cancer Center, New York, NY

${ }^{4}$ Department of Pediatrics, Pharmacology, and Physiology \& Biophysics, Weill Cornell Medical College, Cornell University, New York, NY

*To whom correspondence should be addressed. Email: kentsisresearchgroup@gmail.com 


\section{Supporting Information}

\section{Table of Contents}

Figure S-1. Cross-linked peptide properties across fragmentation methods.

Figure S-2. Cross-linked peptide properties across different proteases

Figure S-3. Comparison of cross-links and cross-link score with published data

Table S-1. List of cross-linked chromatin proteins page S3

page $\mathrm{S} 4$

page $\mathrm{S} 5$

page S6 


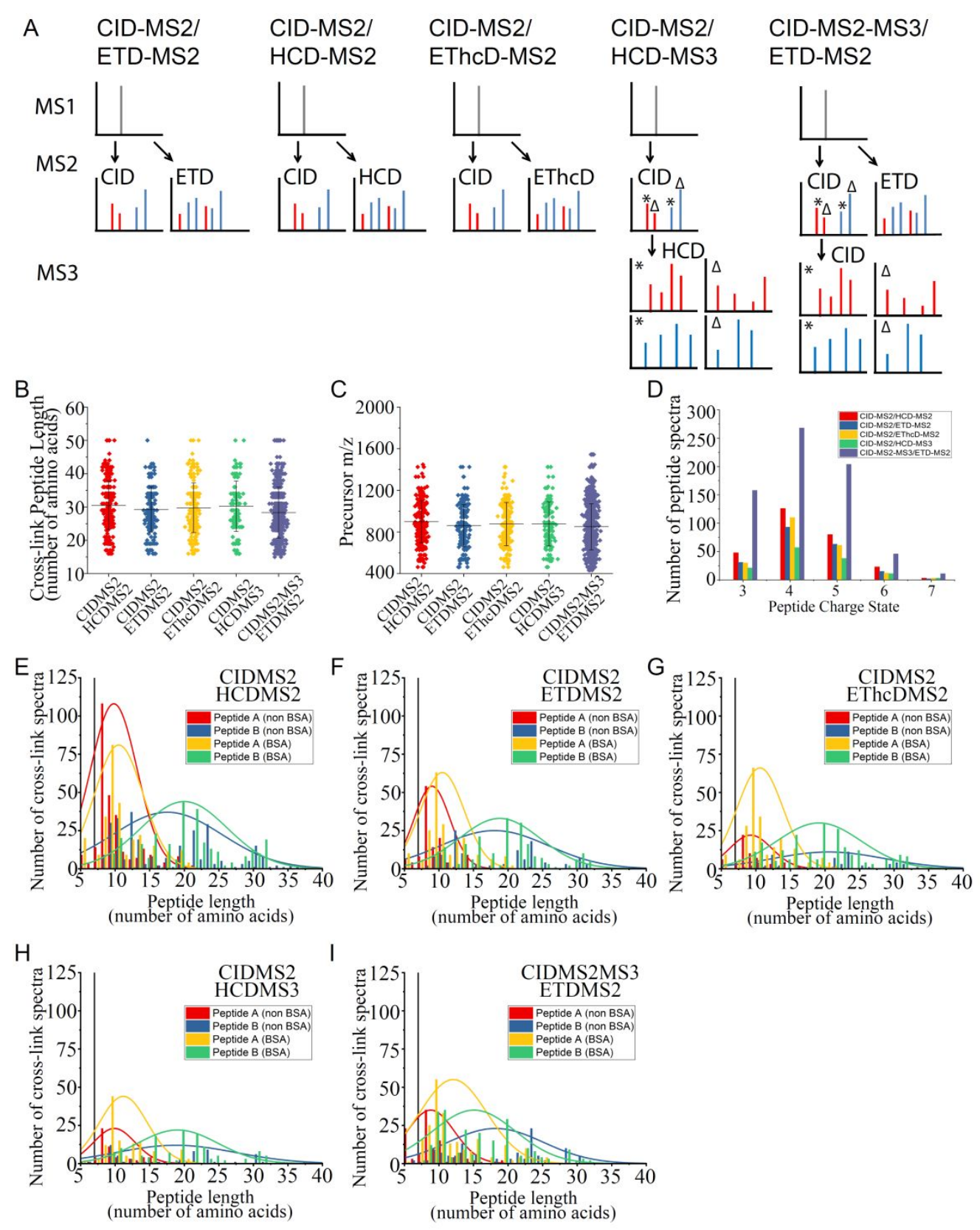

Figure S-1. Characterization of peptide length, $\mathrm{m} / \mathrm{z}$ and charge state of cross-linked peptides from comparison of fragmentation methods: CID-MS2/HCD-MS2, CID-MS2/ETD-MS2, CIDMS2/EThcD-MS2, CID-MS2/HCD-MS3 and CID-MS2-MS3/ETD-MS2. Data shown from cross-linked BSA peptides spiked into non-cross-linked proteome background peptides. A) Schematic of 5 different fragmentation methods. B) Cross-link BSA peptide length by number of amino acids from cross-linked BSA peptide spectra. C) Precursor $\mathrm{m} / \mathrm{z}$ values of cross-linked BSA peptide spectra D) Number of cross-linked BSA peptide spectra based on charge state. E,F,G,H,I) Distribution of peptide A and peptide B lengths for identified cross-linked BSA peptides and cross-linked peptides between non-BSA proteins by CID-MS2/HCD-MS2, CIDMS2/ETD-MS2, CID-MS2/EThcD-MS2, CID-MS2/HCD-MS3 or CID-MS2-MS3/ETD-MS2. Straight vertical line indicates peptide length of 7. 

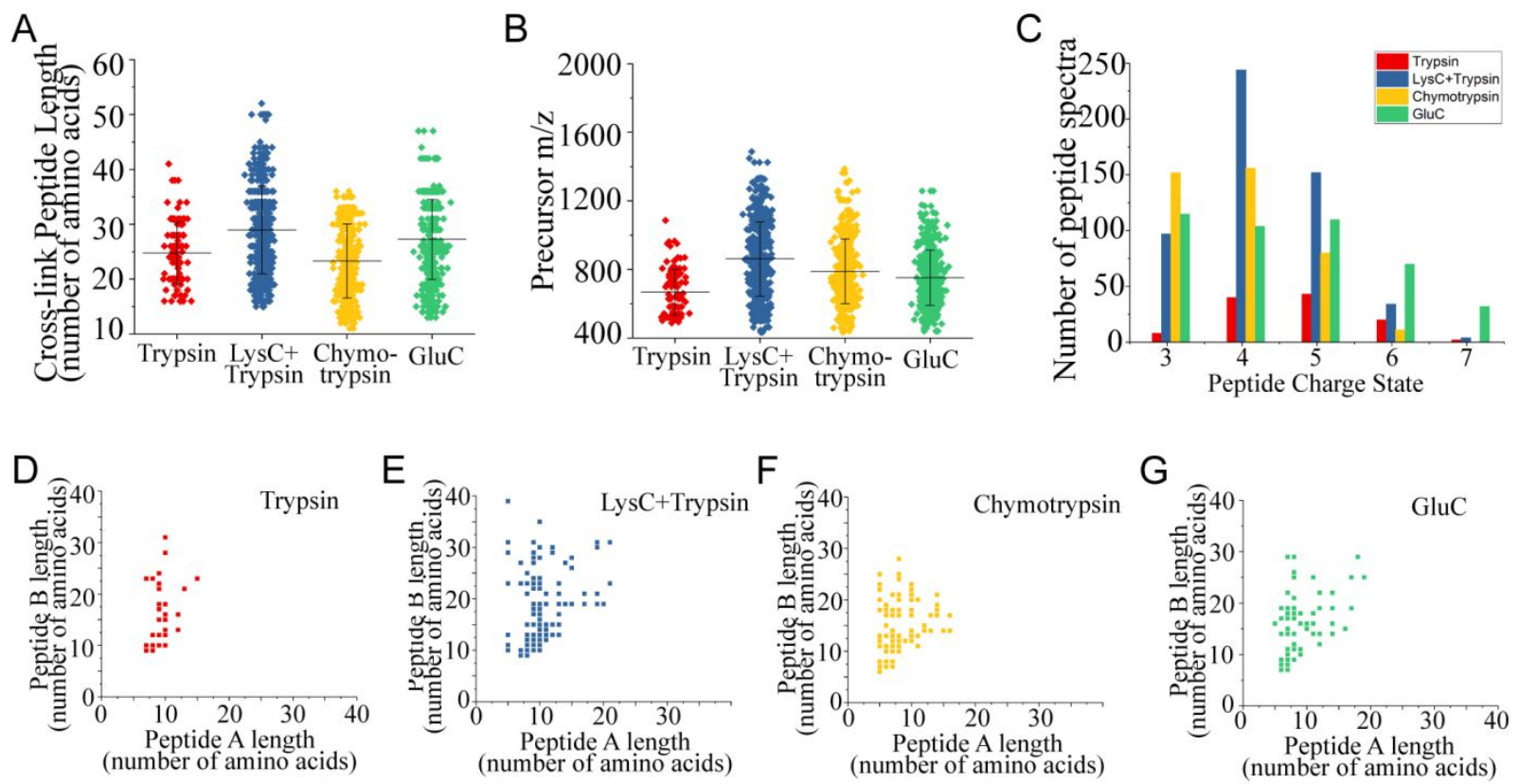

Figure S-2. Characterization of peptide length, $\mathrm{m} / \mathrm{z}$ and charge state of cross-linked peptides from comparison of multiple protease digestion: Trypsin only, combined LysC and Trypsin, chymotrypsin only, GluC only, as analyzed using CID-MS2/HCD-MS2 fragmentation. A) Crosslink peptide length by number of amino acids from cross-linked BSA peptide spectra. B) Precursor $m / z$ value. C) Number of cross-linked peptide spectra based on charge state. D,E,F,G) Comparison of peptide A and peptide B length from cross-linked peptides identified by trypsin only, combined LysC and trypsin, chymotrypsin only or GluC only protease digestion. 

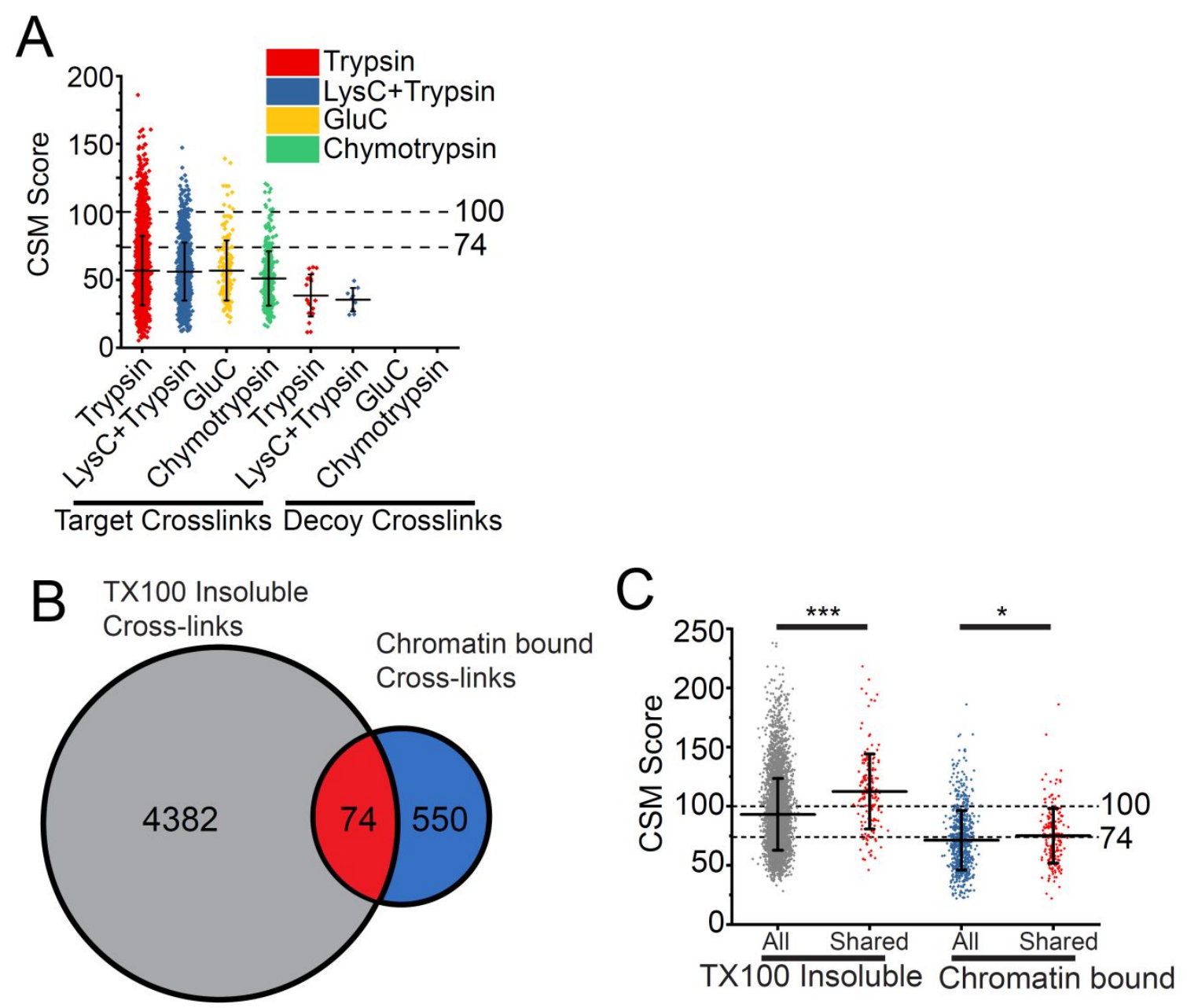

Figure S-3. Comparison of chromatin cross-links identified with published data on cross-linked triton X-100 insoluble nuclear proteins (PMID: 30021884). A) Distribution of CSM score for all target and decoy cross-links identified from chromatin cross-links. 0 decoy cross-links were identified in gluC and chymotrypsin digests of cross-linked chromatin with 1\% FDR specified on Percolator. B) Venn Diagram showing shared cross-link sites identified in triton X-100 insoluble nuclear proteins and purified cross-linked chromatin. C) Distribution of cross-link scores for all triton X-100 insoluble cross-linked peptides and shared cross-linked peptides identified from both triton X-100 insoluble proteins and from chromatin proteins. Error bars represent standard deviation. *student's t-test $p$-value $=0.041, * * *$ student's t-test $p$-value $=3.13^{*} 10^{-8}$. Dashed lines represent CSM score threshold of 100 for $1 \%$ FDR and $74 \%$ for $10 \%$ FDR. 


\section{Supplementary Tables}

Table S-1. List of cross-linked chromatin proteins 